\title{
Avaliação do nível de abertura de dados do governo de Pernambuco a partir da metodologia empregada no Índice de Dados Abertos
}

Elton Rodolfo Assunção da Silva - elton.ras@hotmail.com

Resumo - Dados abertos governamentais contribuem para o fortalecimento da transparência e do controle social e estimulam o desenvolvimento de soluções inovadoras para as demandas sociais. Com vistas a avaliar o nível de abertura de dados governamentais em Pernambuco, de modo a permitir melhor compreensão do ambiente estadual e subsidiar o desenvolvimento de uma política pública que regulamente o tema, ampliando o uso de ferramentas como a Internet e a web para além de portais de dados abertos, foi realizada uma busca na Internet por dados publicados pelos órgãos e entidades do Governo do Estado, orientada pela metodologia empregada na construção do Índice de Dados Abertos para Cidades. Apesar dos resultados evidenciarem o baixo nível de publicação de dados abertos pelo governo do estado, foram encontrados conjuntos disponíveis para a maioria das categorias trabalhadas, sugerindo que o governo já produz e mantém as informações, mas não as publica em formato realmente aberto.

Palavras-chave: Dados abertos governamentais, governo aberto, política pública, tecnologias da informação e comunicação.

\section{Evaluation of the level of data openness of the government of Pernambuco using the methodology used in the Open Data Index}

\begin{abstract}
Open government data contributes to the strengthening of transparency and social control and stimulates the development of innovative solutions for social demands. In order to assess the level of openness of government data in Pernambuco, to allow a better understanding of the state environment and to support a public policy development that regulates the theme, expanding the use of tools such as the Internet and the web in addition to open data portals, an Internet search for data published by the State Government bodies and entities was carried out, guided by the methodology used in the construction of the Open Data Index for Cities. Although the results show low publication level of data opened by the state government, sets were found available for most of the categories worked on, suggesting that the government already produces and maintains the information, but does not publish it in a truly open format.
\end{abstract}

Keywords: Open government data, open government, public policy, information and communication Technologies.

Data da Submissão: 13/01/2021

Data de aceitação: 11/03/2021

1 Secretaria Estadual de Saúde de Pernambuco. 


\section{Introdução}

A abertura de dados governamentais, disponibilidade e acesso as informações geradas pelos órgãos e entidades públicas, sob condições que permitam a reutilização e a redistribuição de maneira universal, sem que que exista discriminação contra áreas de atuação ou contra pessoas ou grupos, possibilita o fortalecimento da transparência, do controle social e da democracia, além de estimular o desenvolvimento de soluções inovadoras para as demandas sociais por entes públicos e privados (OKF, 2020).

Para que esse cenário de dados abertos governamentais (DAG) se concretize, as tecnologias da informação e comunicação (TICs) assumem importante papel, contribuindo com ferramentas como a Internet e a web para a criação de um ambiente onde sistemas e organizações possam atuar de forma colaborativa, combinando diferentes conjuntos de dados e ampliando o engajamento social.

No Brasil, a abertura de dados do governo originou-se a partir da adesão do país a Open Government Partnership (OGP, 2011), onde, em conjunto com outros sete países, foram firmados compromissos relacionados à disponibilização de informações sobre as atividades governamentais, apoio à participação cívica, melhoria nos padrões de integridade profissional dos governos e acesso a novas tecnologias para fins de abertura e accountability (OGP, 2011).

As principais iniciativas de dados abertos governamentais no país correspondem aos portais de dados abertos do Governo Federal e de alguns estados e municípios. Contudo, para que essas iniciativas sejam efetivas, para além de requisitos técnicos que devem ser atendidos pelos conjuntos de dados, como formatos acessíveis e processáveis por máquinas, é fundamental o desenho de uma política robusta capaz de regular os processos de publicação dos dados até o nível das rotinas diárias, levando em consideração os potenciais impactos, em equilíbrio com os receios que órgãos e entidades públicas possam ter em divulgar informações online (NUGROHO, et al., 2015).

Dado que a formulação de uma política pública envolve várias etapas, sendo a primeira delas a concepção, onde são definidas as prioridades e critérios e a negociação com interlocutores úteis ao desenvolvimento da política (técnicos, patrocinadores, associações da sociedade civil e demais parceiros institucionais) (LOPES; AMARAL; CALDAS, 2008), é conveniente e oportuno o conhecimento do cenário atual do tema, fortalecendo as bases para o delineamento de uma política efetiva.

Diante disso, o objetivo desta pesquisa é avaliar o nível de abertura de dados do governo do estado de Pernambuco, de modo a permitir melhor compreensão do ambiente estadual e subsidiar o desenvolvimento de uma política pública que regulamente o tema no Estado.

Para atingir esse objetivo foi realizado um levantamento na Internet por dados publicados pelos órgãos e entidades do governo a partir da metodologia empregada na construção do Índice de Dados Abertos para Cidades edição 2018 (RUEDIGER; MAZOTTE, 2018).

A motivação para esse estudo surgiu a partir da crescente exigência pelo aprimoramento da transparência ativa e acesso à informação e da possibilidade de explorar os dados abertos como meio para o desenvolvimento econômico local. 


\section{Conceitos e trabalhos relacionados}

De acordo com a Open Knowledge Foundation, "dados abertos são dados que podem ser livremente usados, reutilizados e redistribuídos por qualquer pessoa - sujeitos, no máximo, à exigência de atribuição da fonte e compartilhamento pelas mesmas regras" (OKF, 2020).

Conforme apontado por Freitas et al. (2018), a abertura dos dados auxilia na transparência governamental, que, por sua vez, somada à participação cidadã, à accountability e à tecnologia e inovação conformam os princípios de um movimento mais amplo, denominado governo aberto (GA) (OGP, 2011).

Além da divulgação de dados governamentais por meio de elementos de TICs, a materialização do governo aberto depende da definição de padrões, procedimentos e tecnologias para guiar os governos na execução de suas iniciativas, sempre em busca de aumentar a capacidade de interoperabilidade, isto é, a capacidade de integrar diferentes bases de dados permitindo que diversos sistemas e organizações trabalhem juntos (FREITAS et al., 2018; OKF, 2020).

Para que os benefícios proporcionados pelos dados abertos possam ser alcançados, convém ressaltar a forma como eles são disponibilizados à sociedade. Em grande parte das iniciativas a divulgação ocorre por meio de portais na Internet, sobre os quais devem recair considerações para que a descoberta, a extração e o aproveitamento dos dados sejam realizados de modo efetivo (ATTARD et al., 2015).

Nesse sentido, o Open Government Working Group definiu 8 princípios para o livre compartilhamento de dados governamentais, abordando aspectos sobre a natureza do dado e sobre a forma de acesso. São eles: completude, primariedade, temporalidade, acessibilidade, compreensível por máquina, acesso irrestrito e livre de licenças (OGD, 2020; OGWG, 2020).

Já o esquema de cinco estrelas para publicação de dados abertos (BERNERS-LEE, 2010), ilustrado na figura 1, estabelece uma sistemática para avaliar o nível de abertura de dados dos órgãos públicos baseado em um esquema de cinco estágios cumulativos, partindo da mais simples disponibilização na Internet (o que equivale a uma estrela) até o momento em que é possível ter esses dados interligados, configurando o nível mais refinado de dados abertos (cinco estrelas). Quanto mais aberto, maior o número de estrelas para os dados e mais facilidade para ser interligado. 
Figura 1 - Esquema de implementação das 5 estrelas para dados abertos.

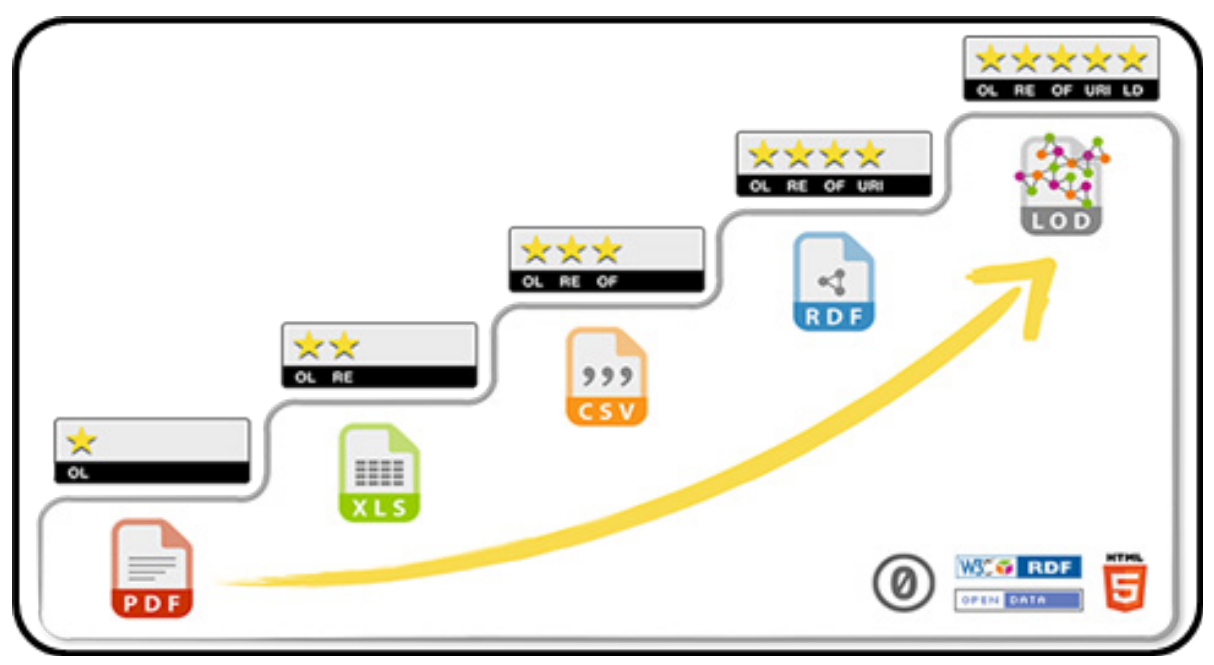

Fonte: Baseado em Berners-Lee (2010).

As estrelas para Dados Abertos podem ser, por ordem de evolução, atribuídas aos conjuntos de dados, conforme descrito no quadro 1, a seguir.

Quadro 1 - Classificação do nível de abertura de dados.

\begin{tabular}{|l|l|}
\hline TOTAL DE ESTRELAS & \multicolumn{1}{c|}{ DESCRIÇÃO } \\
\hline & $\begin{array}{l}\text { Dados disponíveis na Internet (em qualquer formato, como PDF), desde que } \\
\text { com licença aberta, para que seja considerado Dado Aberto. }\end{array}$ \\
\hline & $\begin{array}{l}\text { Dados disponíveis na Internet de maneira estruturada (um arquivo Excel } \mathbb{} \\
\text { com extensão XLS). }\end{array}$ \\
\hline & $\begin{array}{l}\text { Dados disponíveis na Internet, de maneira estruturada, e em formato não } \\
\text { proprietário (CSV ao invés de Excel). }\end{array}$ \\
\hline & $\begin{array}{l}\text { Além de seguir todas as regras anteriores, os dados devem estar dentro dos } \\
\text { padrões estabelecidos pelo W3C (RDF e SPARQL), isto é, usar URL para } \\
\text { identificar coisas e propriedades, de forma que as pessoas possam direcionar } \\
\text { para suas publicações. }\end{array}$ \\
\hline & $\begin{array}{l}\text { Todas as regras anteriores, mais: dados próprios conectados a outros dados, } \\
\text { de forma a fornecer um contexto. }\end{array}$ \\
\hline
\end{tabular}

Fonte: Baseado em Berners-Lee (2010).

As principais propostas para avaliação da maturidade e do nível de abertura dos dados governamentais, assim como recomendações para novas iniciativas, derivam dos oito princípios para dados abertos governamentais e do esquema de cinco estrelas para publicação de dados abertos (ATTARD et al., 2015).

Com relação as categorias de dados que devem ser adotadas em iniciativas de dados abertos, o esforço conjunto do G8 Open Data Charter (2013) reconheceu as categorias apresentadas no quadro 2 como possuindo alto valor por fortalecer a democracia e encorajar a reutilização de dados pelas nações envolvidas. 
Quadro 2 - Categorias de dados de alto valor.

\begin{tabular}{|l|l|}
\hline \multicolumn{1}{|c|}{ CATEGORIAS (ordem alfabética) } & \multicolumn{1}{c|}{ EXEMPLOS DE CONJUNTOS DE DADOS } \\
\hline Empresas & Registro de empresas e negócios \\
\hline Crimes e justiça & Estatísticas criminais, segurança \\
\hline Recursos naturais & $\begin{array}{l}\text { Condições meteorológicas, clima/tempo, agricultura, } \\
\text { silvicultura, caça e pesca }\end{array}$ \\
\hline Educação & $\begin{array}{l}\text { Lista de escolas, desempenho de unidades escolares, } \\
\text { habilidades digitais }\end{array}$ \\
\hline Energia e meio ambiente & Níveis de poluição, consumo de energia \\
\hline Finanças e contratos & $\begin{array}{l}\text { Gastos com transações, contratos, licitações, } \\
\text { orçamentos local e nacional (planejado e gasto) }\end{array}$ \\
\hline Geoespacial & $\begin{array}{l}\text { Topografia, códigos postais, mapas nacionais, mapas } \\
\text { locais }\end{array}$ \\
\hline Desenvolvimento Global & Assistência, segurança alimentar, extrativismo, terras \\
\hline Democracia e accountability & $\begin{array}{l}\text { Relações governamentais, resultados eleitorais, } \\
\text { legislação e estatutos, salários (tabelas salariais), } \\
\text { presentes recebidos }\end{array}$ \\
\hline Saúde & Dados de prescrição, dados de desempenho \\
\hline Ciência e pesquisa & $\begin{array}{l}\text { Dados do genoma, atividades de pesquisa e extensão, } \\
\text { resultados de experimentos }\end{array}$ \\
\hline Estatísticas & $\begin{array}{l}\text { Estatísticas nacionais, dados de censo, infraestrutura, } \\
\text { PIB, ocupação e habilidades }\end{array}$ \\
\hline Mobilidade social e bem-estar & Moradia, seguro saúde, benefícios de desemprego \\
\hline Transporte e infraestrutura & $\begin{array}{l}\text { Horários de transporte público, pontos de acesso, malha } \\
\text { viária }\end{array}$ \\
\hline
\end{tabular}

Fonte: Open Data Charter (2013).

No Brasil, estudos que refletem essas abordagens podem ser observados no Projeto Democracia Digital - Dados abertos nos municípios, estados e governo federal brasileiros (CUNHA et al., 2015) e no Índice de Dados Abertos para Cidades edição 2018 (RUEDIGER; MAZOTTE, 2018).

Por sua vez, a avaliação do grau de transparência de dados abertos governamentais do site dados.rs.gov.br (KLEIN; LUCIANO; MACADAR, 2015) foi realizada a partir do modelo de referência proposto para governo aberto (VELJKOVIĆ; BOGDANOVIĆ-DINIĆ; STOIMENOV, 2014), que utiliza os oito princípios do OGWG para compor o indicador de abertura de dados do governo. Além desse indicador, outros quatro fazem parte da avaliação - existência de categorias mínimas de dados, transparência, participação e colaboração. Como resultado do modelo tem-se duas medidas: o índice de abertura de governo eletrônico, e o nível de maturidade da iniciativa.

Já a avaliação técnica dos conjuntos de dados liberados por capitais brasileiras realizada por Pinho (2017) tomou por base o esquema de cinco estrelas idealizado por Berners-Lee, em conjunto com os oito princípios dos dados governamentais abertos. As nove categorias utilizadas para a avaliação foram selecionadas a partir do que os próprios portais das capitais disponibilizaram, considerando as grandes áreas de formulação de políticas públicas e programas governamentais, como saúde, educação e cultura.

Para além de modelos de avaliação das iniciativas de dados abertos governamentais, a literatura evidencia a necessidade de uma política de abertura de dados robusta, que envolva e 
regulamente não só a publicação de dados, mas o uso, de modo a criar um ecossistema saudável entre publicadores e usuários e facilitar a própria aceitação dos dados abertos (NUGROHO, et al., 2015).

Aspectos técnicos, econômicos, organizacionais e culturais juntam-se aos aspectos legais citados representando elementos críticos que dão origem aos principais desafios, devendo ser considerados para o diagnóstico, implementação e alcance de efetividade das iniciativas de dados abertos (SOLAR, et al., 2014; ATTARD, et al., 2015).

\section{Dados abertos no Brasil e no mundo}

Dados abertos governamentais se apresentam como um dos pilares do movimento de governo aberto, que tem o aumento dos mecanismos de transparência, a reutilização de dados e o contínuo diálogo entre governo e cidadãos como as principais bandeiras.

Apesar do termo "Dados Abertos" ter surgido em 1995, em um documento de uma agência científica americana que abordou a divulgação de dados ambientais e geofísicos, somente em 2007, pensadores e ativistas da Internet se reuniram para definir o conceito de dados abertos públicos ou governamentais (CHIGNARD, 2013).

O conceito apoiou-se, pois, no princípio de que os dados governamentais são propriedades comuns, assim como as ideias científicas, e foi inspirado no movimento do código aberto (open source), que é fundamentado pelos pilares conceituais de abertura, participação e colaboração (ISOTANI; BITTENCOURT, 2015).

Movimentos significativos em direção à abertura de dados podem ser observados no Public Sector Information (PSI) Directive em 2003 na Europa (EUROPEAN PARLIAMENT AND COUNCIL, 2003), na iniciativa para dados abertos do governo norte-americano em 2009 (UNITED STATES, 2009), na Open Government Partnership (OGP, 2011), e no G8 Open Data Charter (2013). Como principais resultados desses movimentos, os portais de dados abertos governamentais, como data.gov.uk, data.gov, e data.gov.sg, fornecem meios para que cidadãos consigam uma série de informações governamentais.

Conforme relatado em Nugroho et al. (2015), algumas nações como Austrália, Dinamarca, Espanha, Reino Unido e Estados Unidos estão em estágio mais avançado no desenvolvimento de políticas para dados abertos, enquanto outros países, como Indonésia e Quênia, demonstram pouco ou nenhum avanço nesse sentido.

No Brasil, projetos como a Lei Complementar n 101/2000 (Lei de Responsabilidade Fiscal) e a Lei Complementar $n^{0}$ 131/2009 (Lei da Transparência), a Carta de Serviços ao Cidadão, o Portal da Transparência e o sítio Comprasnet representam esforços iniciais do governo em aproximar-se do cidadão, incrementando o controle social por meio de tecnologias da informação e comunicação.

Além dessas iniciativas, a adesão do Brasil à Open Government Partnership no ano de 2011, seguida pela publicação da Lei N ${ }^{0} 12.527$ (Lei de Acesso à Informação) e pelos Planos de Ação Nacional de Governo Aberto reforçaram o compromisso com a democracia participativa, 
a promoção de ações e medidas visando o aumento da transparência e do acesso à informação pública, a melhoria na prestação de serviços públicos e com o fortalecimento da integridade pública (OGP, 2011; DUTRA; LOPES, 2013).

Como principais frutos do movimento brasileiro rumo à materialização do governo aberto no país, destacam-se a Arquitetura ePing - Padrões de Interoperabilidade de Governo Eletrônico, conjunto de premissas, políticas e especificações técnicas com o objetivo de possibilitar nível adequado de interoperabilidade entre os serviços disponibilizados pelo governo eletrônico, e a Infraestrutura Nacional de Dados Abertos (INDA) - conjunto de padrões, tecnologias, procedimentos e mecanismos de controle necessários para atender às condições de disseminação e compartilhamento de dados e informações públicas no modelo de Dados Abertos", a Política de Dados Abertos e o próprio Portal de Dados Abertos do Governo Federal (BRASIL, 2015; 2020.).

\section{Metodologia}

Dado o objetivo da pesquisa, que buscou retratar a realidade dos dados abertos em Pernambuco, este trabalho assumiu um caráter essencialmente descritivo - embora em alguns momentos as evidências encontradas tenham sido utilizadas para delinear possíveis relações de causa e efeito. Já a escolha do Estado como lócus da pesquisa foi intencional, considerando as motivações já expostas e a intenção de utilizar os resultados encontrados na prática.

Para que o objetivo pudesse ser alcançado foi adotada a mesma metodologia utilizada para a composição do Índice de Dados Abertos para Cidades edição 2018 (RUEDIGER; MAZOTTE, 2018), dada a riqueza na descrição de como o mesmo foi construído e o grau de detalhamento dos procedimentos que foram executados, permitindo a replicação de grande parte dos processos realizados naquele trabalho neste estudo. O que não ocorreu com outras metodologias que poderiam ter sido utilizadas, a exemplo da empregada no Projeto Democracia Digital - Dados abertos nos municípios, estados e governo federal brasileiros (CUNHA et al., 2015).

De maneira geral, a metodologia demonstrada no Índice de Dados Abertos para Cidades consiste de 17 categorias de dados (ou dimensões) expostas no quadro 3, consideradas por refletirem informações relevantes para a sociedade civil em geral, tendo sido "desenvolvidas de forma colaborativa ao longo dos anos em parceria com especialistas temáticos, incluindo organizações que defendem dados abertos em seus respectivos campos" (RUEDIGER; MAZOTTE, 2018, p. 17).

Quadro 3 - Categorias de dados de referência para avaliação.

\begin{tabular}{|l|l|l|}
\hline \multicolumn{1}{|c|}{ CATEGORIA } & \multicolumn{1}{|c|}{ O QUE SE BUSCA } & CARACTERÍSTICAS REQUERIDAS \\
\hline Orçamento público & $\begin{array}{l}\text { Orçamento do governo municipal. Trata- } \\
\text { se da despesa prevista do governo para o } \\
\text { próximo ano, e não a despesa atual }\end{array}$ & $\begin{array}{l}\text { Orçamento por departamento e } \\
\text { subdepartamento, além de descrições sobre } \\
\text { cada seção do orçamento. Intervalo de } \\
\text { tempo esperado: anual }\end{array}$ \\
\hline Gastos públicos & $\begin{array}{l}\text { Registros dos gastos efetivos do governo } \\
\text { municipal de forma }\end{array}$ & $\begin{array}{l}\text { Departamento que efetuou a transação, data } \\
\text { da transação, identificação do favorecido, } \\
\text { valor nominal da transação. Informações } \\
\text { devem estar disponíveis para cada transação } \\
\text { individual. Intervalo de tempo esperado: } \\
\text { mensal }\end{array}$ \\
\hline
\end{tabular}




\begin{tabular}{|c|c|c|}
\hline Compras públicas & $\begin{array}{l}\text { Todo o processo de execução de uma } \\
\text { compra pública deve ser transparente, } \\
\text { incluindo a fase de licitações e de } \\
\text { concessões. Essa categoria não se } \\
\text { refere ao planejamento de compras } \\
\text { ou à implementação (que faz parte da } \\
\text { categoria gastos públicos) }\end{array}$ & $\begin{array}{l}\text { Na fase de licitação, deve estar disponível } \\
\text { cada licitação por gabinete do governo, } \\
\text { incluindo sua identificação, descrição } \\
\text { e situação. Na fase de concessão, cada } \\
\text { concessão por gabinete do governo deve } \\
\text { estar disponível, incluindo sua identificação } \\
\text { (título), descrição, valor e identificação do } \\
\text { vencedor. Intervalo de tempo esperado: } \\
\text { mensal }\end{array}$ \\
\hline Resultados eleitorais & $\begin{array}{l}\text { Resultados para a última eleição de } \\
\text { governador e deputados. Os dados } \\
\text { eleitorais informam sobre resultados de } \\
\text { votação e processo de votação. Quais } \\
\text { são as maiorias e minorias eleitorais? } \\
\text { Quantos votos são registrados, inválidos } \\
\text { ou nulos? }\end{array}$ & $\begin{array}{l}\text { Resultados para eleições de governador e } \\
\text { deputados devem incluir número de votos } \\
\text { registrados, número de votos inválidos } \\
\text { (brancos/nulos) e urnas danificadas por } \\
\text { seção de votação. Intervalo de tempo } \\
\text { esperado: anual }\end{array}$ \\
\hline Registro de empresas & $\begin{array}{l}\text { Listas de empresas registradas (de } \\
\text { responsabilidade limitada) no Estado. } \\
\text { Essa categoria não precisa incluir dados } \\
\text { financeiros detalhados, como balanços }\end{array}$ & $\begin{array}{l}\text { Nome da empresa, endereço da empresa, } \\
\text { identificador único, com todos os registros } \\
\text { disponíveis para o Estado. Intervalo de } \\
\text { tempo esperado: mensal }\end{array}$ \\
\hline Propriedade da terra & $\begin{array}{l}\text { Mapas com camada de lotes que exibem } \\
\text { limites. Deve haver um registro de } \\
\text { terras com informações sobre parcelas } \\
\text { registradas }\end{array}$ & $\begin{array}{l}\text { Limites e identificação do lote, valor da } \\
\text { propriedade (valor fiscal/venal) e tipo de } \\
\text { posse (público, privado etc). Intervalo de } \\
\text { tempo esperado: anual }\end{array}$ \\
\hline Mapas do estado & $\begin{array}{l}\text { Mapa geográfico do Estado, incluindo } \\
\text { rotas de trânsito, extensões de água e } \\
\text { marcas de relevo. O mapa deve, pelo } \\
\text { menos, ser fornecido em uma escala de } \\
\text { 1: } 250,000(1 \mathrm{~cm}=2,5 \mathrm{~km}) \text {, uma escala } \\
\text { viável para a maioria dos países }\end{array}$ & $\begin{array}{l}\text { Marcações de rotas de trânsito, marcações } \\
\text { de relevo, marcações de extensões de } \\
\text { água e fronteiras do Estado. Para serem } \\
\text { qualificados, os dados devem conter } \\
\text { projeções geográficas que permitam } \\
\text { interpretar as coordenadas. Intervalo de } \\
\text { tempo esperado: anual }\end{array}$ \\
\hline 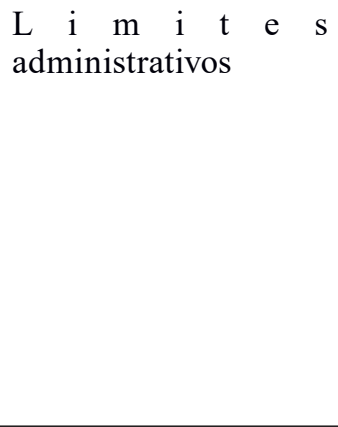 & $\begin{array}{l}\text { Dados sobre unidades administrativas } \\
\text { ou áreas definidas para fins de } \\
\text { administração por um governo (local) }\end{array}$ & $\begin{array}{l}\text { Limite administrativo } 1 \text { (por exemplo: áreas } \\
\text { administrativas), limite administrativo } 2 \\
\text { (por exemplo: bairros), coordenadas de } \\
\text { zonas administrativas } \\
\text { (latitude, longitude), nome do polígono } \\
\text { (departamento, região, cidade) e fronteiras } \\
\text { do polígono. Para serem qualificados, os } \\
\text { dados devem conter projeções geográficas } \\
\text { que permitam interpretar as coordenadas. } \\
\text { Intervalo de tempo esperado: anual }\end{array}$ \\
\hline
\end{tabular}

Continuação

\begin{tabular}{|l|l|l|}
\hline \multicolumn{1}{|c|}{ CATEGORIA } & \multicolumn{1}{|c|}{ O QUE SE BUSCA } & CARACTERÍSTICAS REQUISITADAS \\
\hline Localizações & $\begin{array}{l}\text { Banco de dados de logradouros, } \\
\text { incluindo códigos postais e os locais } \\
\text { correspondentes e relação à latitude e } \\
\text { longitude (ou coordenadas semelhantes } \\
\text { Em um sistema aberto) }\end{array}$ & $\begin{array}{l}\text { CEPs, endereços (obrigatório, se o código } \\
\text { postal não incluir o endereço), coordenadas } \\
\text { (latitude, longitude) em dados disponíveis } \\
\text { para o Estado inteiro. Nota: para serem } \\
\text { qualificados, os dados devem conter } \\
\text { projeções geográficas que permitam } \\
\text { interpretar as coordenadas. Intervalo de } \\
\text { tempo esperado: anual }\end{array}$ \\
\hline
\end{tabular}




\begin{tabular}{|c|c|c|}
\hline $\begin{array}{l}\text { E s t a tís t i c a s } \\
\text { socioeconômicas }\end{array}$ & $\begin{array}{l}\text { Principais estatísticas do Estado sobre } \\
\text { indicadores demográficos e econômicos, } \\
\text { como o produto interno bruto (PIB), } \\
\text { estatísticas de desemprego e população }\end{array}$ & $\begin{array}{l}\text { População do Estado (obrigatório: } \\
\text { informações ou estimativas oficiais } \\
\text { atualizadas todos os anos), produto interno } \\
\text { bruto (medido em preços atuais- constantes, } \\
\text { trimestralmente atualizado - a última } \\
\text { atualização não deve ser há mais de três } \\
\text { meses) e desemprego na cidade (números } \\
\text { absolutos, ou percentuais da população, } \\
\text { atualizados trimestralmente - a última } \\
\text { atualização não deve ser há mais de três } \\
\text { meses) }\end{array}$ \\
\hline Atividade legislativa & $\begin{array}{l}\text { Dados sobre as propostas de leis } \\
\text { discutidas no parlamento estadual, } \\
\text { incluindo votos dos parlamentares. } \\
\text { Não deve ser confundido com as leis } \\
\text { aprovadas. Os dados devem estar } \\
\text { disponíveis para o período atual da } \\
\text { legislatura }\end{array}$ & $\begin{array}{l}\text { Conteúdo da proposta, autor da proposta, } \\
\text { situação da tramitação, votos na proposta } \\
\text { por membro do parlamento estadual, } \\
\text { transcrições dos debates sobre a proposta, } \\
\text { todos disponíveis para a legislatura atual. } \\
\text { Intervalo de tempo esperado: semanal }\end{array}$ \\
\hline Leis do estado & $\begin{array}{l}\text { Dados sobre todas as leis e estatutos } \\
\text { vigentes no âmbito do Estado (não se } \\
\text { trata de informações sobre atividade } \\
\text { legislativa) }\end{array}$ & $\begin{array}{l}\text { Conteúdo da lei e sua situação, data da } \\
\text { última alteração e emendas à lei. Devem } \\
\text { existir informações sobre todas as leis } \\
\text { vigentes no âmbito estadual. Intervalo de } \\
\text { tempo esperado: trimestral }\end{array}$ \\
\hline Qualidade do ar & $\begin{array}{l}\text { Dados sobre a concentração média } \\
\text { diária de poluentes do ar, especialmente } \\
\text { aqueles potencialmente prejudiciais } \\
\text { para a saúde humana. Os dados devem } \\
\text { estar disponíveis para todas as estações } \\
\text { ou zonas de monitoramento do ar no } \\
\text { Estado }\end{array}$ & $\begin{array}{l}\text { Concentração dos seguintes poluentes: } \\
\text { material particulado (MP), óxidos de } \\
\text { enxofre (SOX), óxidos de nitrogênio (NOX), } \\
\text { monóxido de carbono (CO), ozônio (O3), } \\
\text { e compostos orgânicos voláteis (VOCS). } \\
\text { Dados disponíveis por estação/zona de } \\
\text { monitoramento de ar. Intervalo de tempo } \\
\text { esperado: diário }\end{array}$ \\
\hline Qualidade da água & $\begin{array}{l}\text { Dados de qualidade da água por fonte } \\
\text { de água. A categoria de dados considera } \\
\text { a qualidade das fontes de água potável } \\
\text { designadas. Se tais informações não } \\
\text { estiverem disponíveis, a categoria } \\
\text { refere-se a fontes de água ambientais } \\
\text { (lagos, rios, águas subterrâneas) }\end{array}$ & $\begin{array}{l}\text { Concentração das seguintes substâncias: } \\
\text { coliformes fecais, arsênico, flúor, nitratos e } \\
\text { sólidos dissolvidos totais. Os dados devem } \\
\text { estar disponíveis por fonte de água para } \\
\text { todo o Estado. Intervalo de tempo esperado: } \\
\text { semanal }\end{array}$ \\
\hline Escolas públicas & $\begin{array}{l}\text { Dados sobre matrícula e localização } \\
\text { de escolas públicas para toda a cidade, } \\
\text { incluindo todos os níveis (ensino } \\
\text { infantil, fundamental e médio) e todo } \\
\text { tipo de administração (federal, estadual } \\
\text { e municipal) }\end{array}$ & $\begin{array}{l}\text { Número de matrículas por escola, } \\
\text { coordenadas (endereço ou latitude / } \\
\text { longitude). Dados disponíveis por escola } \\
\text { pública e todos os níveis da educação } \\
\text { básica. Intervalo de tempo esperado: anual }\end{array}$ \\
\hline
\end{tabular}

Continuação

\begin{tabular}{|l|l|l|}
\hline \multicolumn{1}{|c|}{ CATEGORIA } & \multicolumn{1}{|c|}{ O QUE SE BUSCA } & CARACTERÍSTICAS REQUISITADAS \\
\hline Transporte público & $\begin{array}{l}\text { Informações sobre o horário dos } \\
\text { transportes públicos, itinerários, } \\
\text { estações/paradas e ciclofaixas para todo } \\
\text { o Estado. Devem ser apresentados para } \\
\text { cada meio de transporte (ônibus, metrô, } \\
\text { trem etc.) e para cada linha e parada/ } \\
\text { transporte, cronograma de linha para cada } \\
\text { parada/estação, itinerários para cada linha } \\
\text { de todos os modos de transporte público, } \\
\text { cartografia de ciclofaixas/ciclovias para } \\
\text { todo o Estado. Os dados devem mostrar } \\
\text { conexões entre diferentes modais de } \\
\text { transporte. Intervalo de tempo esperado: } \\
\text { mensal }\end{array}$ \\
\hline
\end{tabular}




\begin{tabular}{|c|c|c|}
\hline Estatísticas do crime & $\begin{array}{l}\text { Indicadores básicos sobre crime como } \\
\text { registros de assaltos, homicídios, } \\
\text { estupros e apreensão de armas de } \\
\text { fogo, disponíveis para cidades por } \\
\text { bairro ou divisão equivalente. Para as } \\
\text { armas de fogo, os dados devem incluir } \\
\text { a classificação da apreensão - tipo de } \\
\text { arma e calibre - e se é legal ou ilegal }\end{array}$ & $\begin{array}{l}\text { Número de assaltos, número de homicídios, } \\
\text { número de estupros e apreensão de armas. } \\
\text { Dados disponíveis por bairro ou equivalente. } \\
\text { Intervalo de tempo esperado: semestral. }\end{array}$ \\
\hline
\end{tabular}

Fonte: Baseado em Ruediger; Mazotte (2018).

As categorias orientam as buscas na Internet por conjunto de dados que devem atender a algumas características também pré-estabelecidas. Na categoria 'gastos públicos', por exemplo, os dados encontrados devem conter informações relacionadas ao departamento do governo que efetuou a transação, data da transação, identificação do favorecido e o valor nominal da transação, dentro do intervalo de tempo mensal.

A partir do momento em que se encontram dados que satisfaçam minimamente as características requisitadas, o conjunto é avaliado usando uma série de questões que examinam a abertura dos conjuntos de dados com base na Definição de Abertura - Open Definition - e na Carta de Dados Abertos - Open Data Charter, constantes em um mapa construído com as categorias de dados, questões verificadoras, pontuações atribuídas e características requisitadas.

Para ilustrar, algumas perguntas são ‘É possível baixar os dados de uma só vez?' e ‘Os dados estão em formato de arquivo aberto e processáveis por máquina?’.

Cada pergunta do questionário mede um aspecto legal, técnico ou prático relevante da abertura de dados e possui uma pontuação específica, se atendida. A soma da pontuação de todas as perguntas totaliza 100 (cem), valor máximo que cada categoria de dados pode alcançar na avaliação.

As etapas de operacionalização da pesquisa estão apresentadas na figura 2.

Figura 2 - Protocolo de operacionalização da pesquisa.

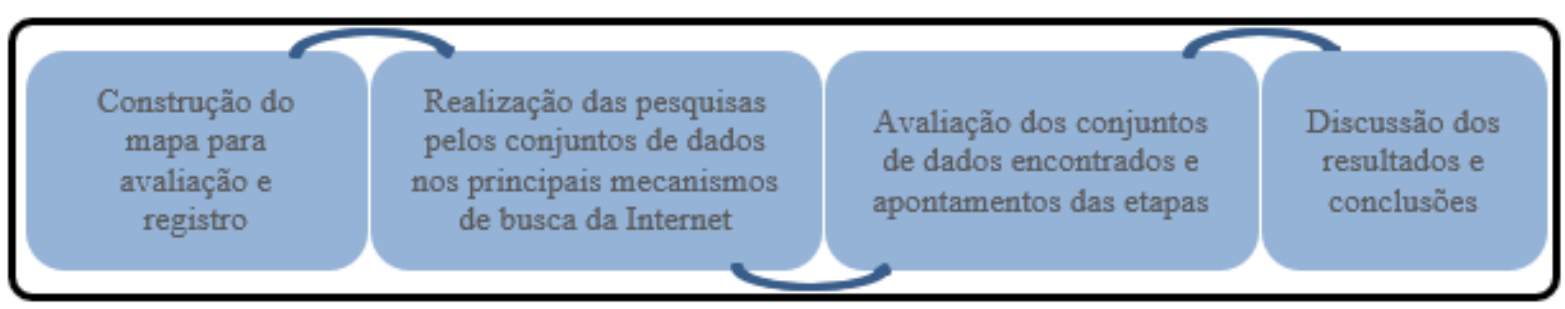

Fonte: Elaboração própria.

Neste estudo, as buscas na Internet ocorreram entre os dias 05/01/2020 e 20/03/2020 e uma revisão, com o objetivo de identificar incoerências na avaliação das categorias, ocorreu entre 23 e 29/03/2020.

\section{Resultados e discussão do levantamento}

As pontuações obtidas em cada dimensão permitiram estabelecer uma distribuição que reflete o nível de abertura de dados em cada uma das categorias de dados, conforme representada na figura 3. 
Figura 3 - Mapa da pontuação alcançada por cada categoria.

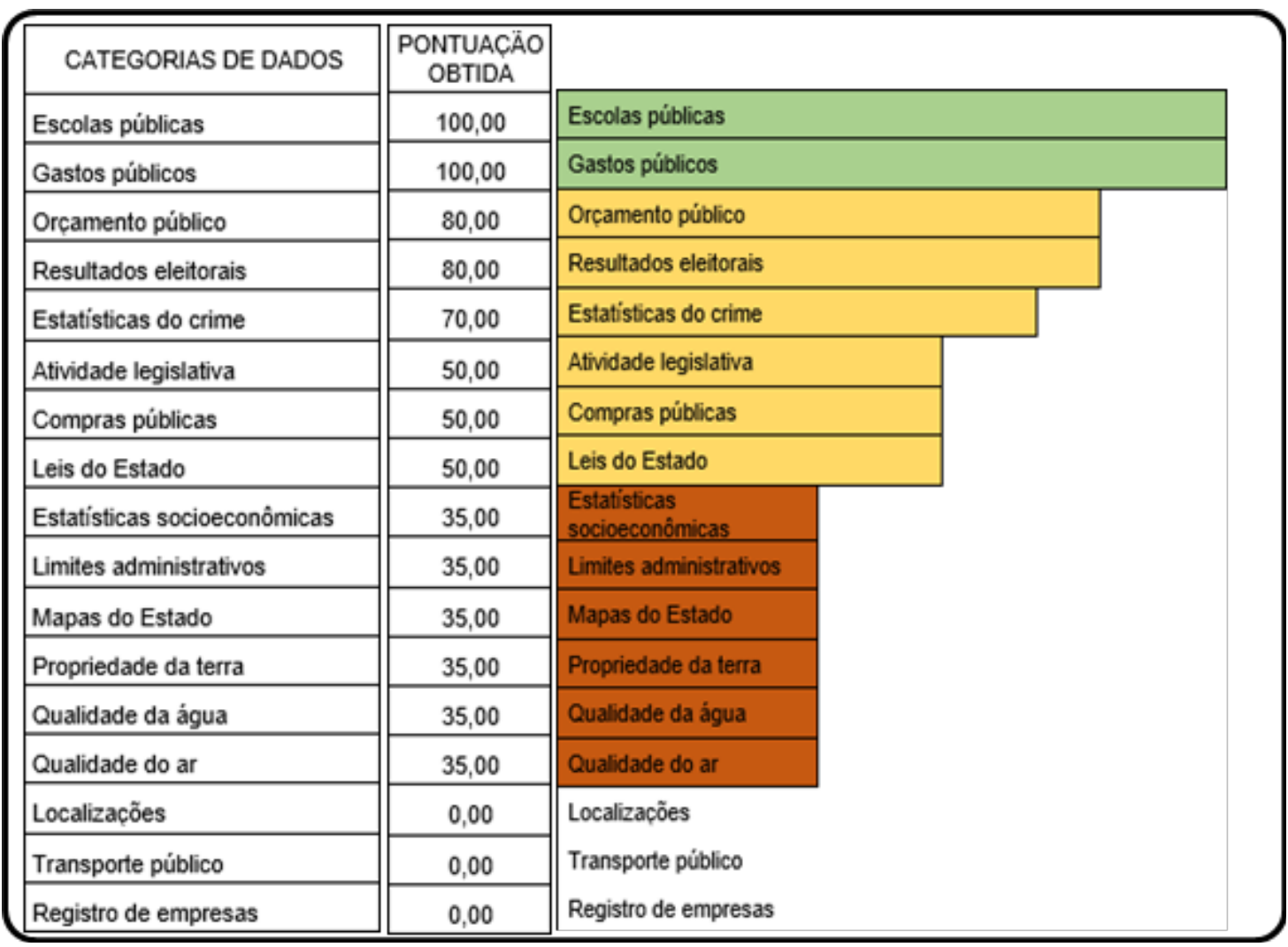

Fonte: Elaboração própria.

A partir da análise da figura 3, é possível classificar as categorias de dados em dados abertos; dados públicos, mas não plenamente abertos; e ausência de dados. A seguir, cada uma das categorias será discutida no interior desses grupos.

\subsection{Dados abertos}

O agrupamento em dados abertos compreende as categorias que alcançaram nota $100 \%$, nas quais os dados atenderam aos requisitos de legibilidade por máquina, formatos abertos, pleno acesso, disponíveis de forma completa, sem custo e juridicamente em domínio público. As únicas categorias que alcançaram a nota máxima foram Gastos públicos e Escolas públicas.

Encontrar uma base de dados atualizada e que apresentasse minimamente as características desejadas para a categoria Gastos públicos foi relativamente fácil para o pesquisador, bastando uma consulta pelo próprio nome da categoria no Google. O segundo resultado da busca conduziu ao Portal da Transparência do Governo de Pernambuco, no qual foi possível encontrar uma seção dedicada as despesas detalhadas, com possibilidade de exportar uma planilha com os dados em formato .XLS, e um menu de apoio de dados abertos, onde é possível localizar o conjunto de dados desejados em formato .CSV.

Aqui cabe destacar os resultados encontrados por Cunha et al. (2015) onde os dados orçamentários e financeiros foram os mais divulgados em formato aberto, refletindo, provavelmente, a Lei de Responsabilidade Fiscal e a Lei da Transparência, corroborando o cenário de priorização desses tipos de dados impelido pela legislação, sugerido por Sucha, Grönlund e Janssen (2015). 
Ao passo em que esta priorização dá indícios de avanços em termos de transparência, abre espaço para outros argumentos para o uso de dados abertos, como o estímulo à economia pelos negócios, a prestação de serviços por parte de empresas (SUCHA; GRÖNLUND; JANSSEN, 2015).

Já a busca pelos dados na categoria Escolas públicas iniciou-se pela navegação no Sistema de Informações da Educação de Pernambuco (SIEPE) e foi verificado que as informações disponibilizadas na consulta por escola atendiam as características requisitadas para qualificação da base de dados. Contudo, dada a impossibilidade de baixar/exportar os dados e a ausência da última data de atualização das informações, a pesquisa continuou através da plataforma disponibilizada pelo INEP que apresentou as informações contendo as características requeridas e permitiu que a base de dados fosse exportada/baixada (a base de dados do INEP foi qualificada para continuidade do levantamento).

\subsection{Dados públicos, mas não plenamente abertos}

Nesse grupo se enquadram as categorias que apresentam dados públicos, mas com alguma limitação que não os fazem plenamente abertos, como estarem em formato fechado para edição, como .PDF, ou desatualizados, com alguma limitação de acesso (necessidade de cadastro ou proteção por patente ou algo do gênero), disponíveis apenas parcialmente, ou sem possibilidade de download. As pontuações para essa classificação são intermediárias, entre 0 e $100 \%$ e neste levantamento representam $70 \%$ das categorias avaliadas.

As categorias Orçamento público, Resultados eleitorais e Estatísticas do crime obtiveram boas pontuações, mas apresentaram alguns gargalos. Embora a busca por dados do orçamento público estadual, por exemplo, tenha sido fácil, dada a necessidade de aprovação do mesmo por meio de Lei e publicação em canais oficiais, a base completa encontrada estava em formato .PDF, limitando o processamento por pelo menos um software livre e ferramenta de código aberto, sendo consideradas bases distintas para as receitas e as despesas que, integradas, conformam o orçamento por inteiro. Situação semelhante foi encontrada com os arquivos da categoria Resultados eleitorais, dado que estavam compactados, exigindo que o usuário possua algum descompactador de arquivos.

Já na categoria Estatísticas do crime, a base de dados avaliada atendeu às características mínimas requisitadas (número de assaltos, número de homicídios, número de estupros e apreensão de armas), mas também estavam e formato .PDF e sem licença explícita ou declaração sobre os termos de uso dos dados que deixasse claro seu domínio público ou qualquer referência explícita a algum dispositivo legal que fornecesse as bases para a divulgação da informação.

Por sua vez, as categorias Atividade legislativa, Leis do Estado e Compras públicas alcançaram 50 pontos, cada. Para todas as bases encontradas nessas categorias, não foi identificada a possibilidade de download nem a existência de licença explícita ou declaração sobre os termos de uso dos dados e os dados não estavam em formato de arquivo aberto e processáveis por máquina.

As categorias Estatísticas socioeconômicas, Limites administrativos, Mapas do Estado, Propriedade da terra, Qualidade da água e Qualidade do ar obtiveram apenas 35 pontos na avalição. Além de não ser possível realizar o download das bases, da não identificação de licença explícita ou declaração sobre os termos de uso dos dados e dos dados que não estavam em 
formato de arquivo aberto e processáveis por máquina, outro gargalo surgiu para esse grupo: a impossibilidade de verificar a última data de atualização dos dados ou as informações correspondiam a intervalos de tempo considerados desatualizados.

Convém destacar a dificuldade encontrada durante a busca de dados dentro da categoria Propriedade da terra. Os primeiros resultados em pesquisa no Google retornaram notícias relacionadas ao programa de regularização fundiária de Pernambuco. A partir daí foram identificados três órgãos que poderiam apresentar os dados desejados: o Instituto de Terras e Reforma Agrária do Estado (ITERPE), a Companhia Estadual de Habitação e Obras (CEHAB) e a Pernambuco Participações e Investimentos S.A. (PERPART). Foram encontrados dados mais próximos às características desejadas no site do ITERPE.

Essa dificuldade pode ser explicada pelo fato de a maior parte dos dados de propriedade de terra serem disponibilizados para fins de cobrança de IPTU, o que faz sentido na dimensão municipal.

\subsection{Ausência de dados}

A classificação neste grupo é destinada às categorias em que não foram localizados dados pela não produção por parte do governo ou pela falta de divulgação. Naturalmente, a pontuação obtida por essas categorias foi zero. Neste levantamento não foram encontradas bases de dados para as categorias Localizações, Transporte público e Registro de empresas.

A pesquisa por bases de dados da categoria Localizações foi bastante difícil pela falta de termos claros de pesquisa e pela própria ausência de bases que atendessem as características desejadas e pudessem ser qualificadas para análise. Foram feitas pesquisas no Google usando os termos "localizações pernambuco", "endereços pernambuco", "códigos postais pernambuco", "coordenadas geográficas pernambuco" e outras variações na mesma linha, sem que os resultados fossem adequados a esta pesquisa. Foi realizada uma consulta diretamente no site dos Correios e no Portal de Dados Abertos do Governo Federal, ambas sem sucesso na identificação de bases de dados relacionadas ao tema.

Revisitando os resultados do trabalho que deu origem a esta pesquisa, notou-se que esta categoria apresentou uma série de gargalos entre as cidades objeto do estudo. Lá, foi constatado que conjuntos de dados semelhantes, que poderiam satisfazer as categorias Limites Administrativos, Mapas da Cidade e Localizações, foram extraídos de plataformas centralizadas, que congregavam diferentes conjuntos de dados de forma integrada em um único portal, apoiados em tecnologias de georreferenciamento.

De fato, essa era a expectativa durante as buscas, dado que foram encontrados mapas dinâmicos a partir dos quais foram analisadas as categorias mapas do Estado e Limites administrativos, o que não se concretizou. Com efeito, iniciativas como estas representam importante avanço na divulgação de informações e são consideradas boas práticas de transparência.

No tocante à categoria Transporte público, foram encontrados dados relacionados apenas à Região Metropolitana do Recife. Apesar de as informações encontradas nas buscas atenderem a algumas das características desejadas, essa limitação associada a não apresentação de cartografia de ciclofaixas/ciclovias no Estado nem as conexões entre diferentes modais de transporte (não fica claro se existem outros modais além do rodoviário), impossibilitou a quali- 
ficação como uma base de dados para continuidade da verificação.

Pontua-se que a metodologia aqui empregada foi delineada para levantamentos no universo municipal, sendo razoável admitir a dificuldade para obtenção das informações no âmbito estadual. Foi identificada na estrutura do Poder Executivo de Pernambuco a Empresa Pernambucana de Transporte Intermunicipal (EPTI), que tem por objeto social a "gestão do Sistema de Transporte Coletivo Intermunicipal de Passageiros do Estado de Pernambuco STIP, envolvendo o planejamento, a implementação, a fiscalização e a outorga a terceiros dos serviços a ele relacionados" (PERNAMBUCO, 2019). Mas consultando o site oficial da instituição também não foram localizadas as informações.

Por fim, quanto à categoria Registro de empresas, destaca-se que inicialmente um determinado conjunto de dados foi considerado apto para ser avaliado, mas durante a fase de revisão do levantamento, foi identificada que a base não atendia minimamente as características requeridas (nome da empresa, endereço da empresa, identificador único, com todos os registros disponíveis para o Estado). A pesquisa seguiu nos sites do IBGE e do SEBRAE, sem sucesso. Dessa forma, como não foi encontrada nenhuma outra base/conjunto de dados adequados, a categoria teve a pontuação zerada.

\subsection{Avaliação geral}

A partir da avaliação de cada categoria de dados foi possível obter o escore, que mensura a adequação dos dados disponibilizados pelo governo aos critérios de transparência utilizados em diversos países do mundo e é dado por uma pontuação objetiva. Foi possível também verificar o "\%open", que calcula qual o percentual dos conjuntos de dados avaliados que atende a todos os critérios da metodologia. O quadro 4 apresenta o resultado das duas métricas para o Estado de Pernambuco.

Quadro 4 - Avaliação geral do nível de abertura de dados no Estado de Pernambuco.

\begin{tabular}{|l|c|c|}
\hline \multirow{2}{*}{ PERNAMBUCO } & ESCORE & \%OPEN \\
\cline { 2 - 3 } & 46,47 & 13,33 \\
\hline
\end{tabular}

Fonte: Elaboração própria.

Esse resultado demonstra que o Estado de Pernambuco precisa superar alguns desafios para avançar na abertura de dados governamentais. Significa que existem gargalos que dificultam a aproximação dos conjuntos de dados públicos aos critérios da Open Definition, dado que a pontuação média entre as categorias não alcançou 50\%.

Nota-se, ainda, a falta de efetividade em divulgar bases 100\% abertas, apenas 2 categorias o fizeram e, em uma delas (Escolas públicas), a base foi obtida a partir de um órgão do governo federal. Uma pontuação alta na métrica "\%open" reflete a intenção do governo em conceder à população dados abertos de qualidade, atendendo tanto a necessidade de pesquisadores e desenvolvedores que queiram trabalhar com essas bases quanto de cidadãos comuns, que desejem informações sobre seu governo, políticas públicas, leis, local onde vivem etc.

Os principais gargalos constatados ao longo do levantamento e as categorias os apresentaram estão apresentados no quadro 5. 
Quadro 5-Gargalos por categoria de dados.

\begin{tabular}{|c|c|c|c|c|c|}
\hline CATEGORIAS & $\begin{array}{c}\text { ATENDIMENTO } \\
\text { DAS CARACTE- } \\
\text { RÍSTICAS } \\
\text { DESEJADAS }\end{array}$ & $\begin{array}{c}\text { IMPOSSIBILIDADE } \\
\text { DE BAIXAR OS } \\
\text { DADOS DE UMA } \\
\text { SÓ VEZ }\end{array}$ & $\begin{array}{l}\text { ATUALI- } \\
\text { ZAÇÃO DOS } \\
\text { DADOS }\end{array}$ & $\begin{array}{l}\text { USABILIDADE } \\
\text { JURÍDICA } \\
\text { (LICENÇA } \\
\text { ABERTA/DO- } \\
\text { MÍNIO PÚBLICO) }\end{array}$ & $\begin{array}{l}\text { FORMATO } \\
\text { DE ARQUIVO } \\
\text { ABERTOE } \\
\text { PROCES- } \\
\text { SÁVEIS POR } \\
\text { MÁQUINA }\end{array}$ \\
\hline $\begin{array}{l}\text { O R C A A M E N T O } \\
\text { PÚBLICO }\end{array}$ & & $\mathrm{X}$ & & & \\
\hline \multicolumn{6}{|l|}{ GASTOS PÚBLICOS } \\
\hline COMPRAS PÚBLICAS & & $\mathrm{X}$ & & $\mathrm{X}$ & $\mathrm{X}$ \\
\hline $\begin{array}{l}\text { R E S U L T A D O S } \\
\text { ELEITORAIS }\end{array}$ & & $\mathrm{X}$ & & & \\
\hline $\begin{array}{l}\text { PROPRIEDADE DA } \\
\text { TERRA }\end{array}$ & & $\mathrm{X}$ & $\mathrm{X}$ & $\mathrm{X}$ & $\mathrm{X}$ \\
\hline MAPAS DO ESTADO & & $\mathrm{X}$ & $\mathrm{x}$ & $\mathrm{X}$ & $\mathrm{x}$ \\
\hline $\begin{array}{lllllll}\mathrm{L} & \mathrm{I} & \mathrm{M} & \mathrm{I} & \mathrm{T} & \mathrm{E} & \mathrm{S} \\
\text { ADMMINISTRATIVOS }\end{array}$ & $\mathrm{X}$ & $\mathrm{X}$ & $\mathrm{X}$ & $\mathrm{X}$ & $\mathrm{X}$ \\
\hline $\begin{array}{l}\text { E S T A T Í S T I C A S } \\
\text { SOCIOECONÔMICASS }\end{array}$ & & $\mathrm{X}$ & $\mathrm{X}$ & $\mathrm{X}$ & $\mathrm{X}$ \\
\hline $\begin{array}{l}\text { A T I V I D A D E } \\
\text { LEGISLATIVA }\end{array}$ & $\mathrm{X}$ & $\mathrm{X}$ & & $\mathrm{X}$ & $\mathrm{X}$ \\
\hline LEIS DO ESTADO & & $\mathrm{X}$ & & $\mathrm{x}$ & $\mathrm{x}$ \\
\hline QUALIDADE DO AR & & $\mathrm{X}$ & $\mathrm{X}$ & $\mathrm{X}$ & $\mathrm{X}$ \\
\hline $\begin{array}{l}\text { QUALIDADE DA } \\
\text { AGUA }\end{array}$ & & $\mathrm{X}$ & $\mathrm{X}$ & $\mathrm{X}$ & $\mathrm{X}$ \\
\hline \multicolumn{6}{|l|}{ ESCOLAS PÚBLICAS } \\
\hline $\begin{array}{l}\text { ESTATÍSTICAS DO } \\
\text { CRIME }\end{array}$ & & & & $\mathrm{X}$ & $\mathrm{X}$ \\
\hline
\end{tabular}

Fonte: Elaboração própria.

\section{Considerações finais}

O objetivo do artigo de avaliar o nível de abertura de dados do governo do estado de Pernambuco, de modo a permitir melhor compreensão do ambiente estadual e subsidiar o desenvolvimento de uma política pública que regulamente o tema no Estado foi atingido por meio da utilização da metodologia empregada na construção do Índice de Dados Abertos para Cidades e da execução das etapas previstas no protocolo de operacionalização da pesquisa.

A revisão da literatura evidenciou a importância do desenvolvimento de uma política de dados abertos que enderece as preocupações dos órgãos e entidades públicos, estabeleça com clareza os processos a serem realizados e estimule tanto a divulgação quanto o uso dos dados. Para que isso seja possível é crucial que se conheça o que está sendo praticado, os principais envolvidos com a produção e divulgação de dados e as dificuldades existentes.

A partir da análise dos resultados constatou-se o baixo nível de publicação de dados abertos no Estado de Pernambuco. Apesar disso, é factível que o nível de abertura de dados seja melhorado uma vez que para a maioria das categorias de dados foram encontrados conjuntos disponíveis, sugerindo que o governo já produz as informações e as mantém de alguma forma, embora não realize a divulgação em formato realmente aberto.

Destaca-se que, quando das buscas pelos conjuntos de dados na Internet, frequente- 
mente surgiram entre os resultados das pesquisas o Anuário Estatístico de Pernambuco e a Base de Dados do Estado (BDE) ${ }^{3}$, ambos mantidos pela Agência Estadual de Planejamento e Pesquisas de Pernambuco (CONDEPE / FIDEM), evidenciando que o Estado pode ter uma estrutura organizacional e um banco de dados razoavelmente adequados para a implementação de uma iniciativa de dados abertos, cabendo ajustes em termos de processos e adequações aos princípios e requisitos técnicos apresentados.

Como limitações desta pesquisa cita-se o fato de a metodologia escolhida ter sido delineada para análise de cidades. Isso fica mais claro quando considerado que a maior parte dos dados relacionados à propriedade de terra são identificados para fins de cobrança de IPTU, sendo adequada sua análise no âmbito municipal. Após discussão com um dos pesquisadores envolvidos na construção do Índice de Dados Abertos para Cidades, concluiu-se que, para a avaliação de outras esferas, a categoria não deveria ser necessariamente desconsiderada, mas precisaria ser repensada, com outras características, por exemplo. Aqui a opção foi pela manutenção da categoria.

Os principais estudos relacionados com esta pesquisa apresentam metodologias de análise que trazem categorias de dados pré-definidas, que é importante para estabelecer uma referência para as iniciativas de dados abertos. Contudo, convém incluir formas de avaliar se realmente algumas categorias são relevantes nas avaliações de determinadas localidades, tendo em vista as limitações de recursos observadas nos governos e o sobre o que de fato a sociedade demonstra interesse.

Além de fornecer insumos para o desenvolvimento de uma política de dados abertos, evidenciando o caráter prático deste trabalho por meio de um retrato da atual situação do Estado, e das contribuições direcionadas à metodologia, as implicações para a academia estendem-se para a abordagem da temática a partir de teorias capazes de analisar o fenômeno sob a perspectiva institucional, uma vez que existiram outras iniciativas relacionadas a dados abertos no âmbito do Poder Executivo em Pernambuco que não tiveram continuidade.

Diante disso, é conveniente e oportuno melhorar a compreensão das razões pelas quais as iniciativas foram interrompidas, relacionando ao que Silva (2018) explorou em termos de continuidades, inovações e retrocessos na evolução do governo digital em Pernambuco.

2 http://www.anuario.pe.gov.brl.

3 http://www.bde.pe.gov.br/estruturacaogeral/conteudo_site2.aspx. 


\section{Referências}

ATTARD, J. et al. A systematic review of open government data initiatives. Government Information Quarterly, v. 32, n. 4, p. 399-418, 2015. Disponível em https://www.researchgate. net/profile/Judie_Attard/publication/281349915 A Systematic Review of Open Government_Data_Initiatives/links/55e575de08aec74dbe7327ef/A-Systematic-Review-ofOpen-Government-Data-Initiatives.pdf. Último acesso em: 05 abr. 2020.

BERNERS-LEE, T. Open, Linked Data for a Global Community. Gov 2.0 Expo. Cidade: Washington. 2010.

BRASIL. Ministério do Planejamento, Orçamento e Gestão. Guia de Interoperabilidade: Cartilha Técnica / Ministério do Planejamento, Orçamento e Gestão. - Brasília: MP, 2015. Disponível em http:/www.gespublica.gov.br/sites/default/files/documentos/manual do gestor de interoperabilidade de governo eletronico.pdf. Último acesso em: 28 abr. 2020.

BRASIL. Infraestrutura Nacional de Dados Abertos. 2020. Disponível em https://wiki.dados. gov.br/. Último acesso em: 30 out. 2020.

BRASIL. Lei complementar $n^{0}$ 101, de 4 de maio de 2000. Estabelece normas de finanças públicas voltadas para a responsabilidade na gestão fiscal e dá outras providências. BRASILIA, 5 maio 2000. Disponível em: http://www.planalto.gov.br/ccivil 03/leis/lcp/lcp101.htm. Último acesso em: 10 jan. 2021.

BRASIL. Lei complementar $\mathbf{n}^{\circ}$ 139, de 27 de maio de 2009. Acrescenta dispositivos à Lei Complementar no 101, de 4 de maio de 2000, que estabelece normas de finanças públicas voltadas para a responsabilidade na gestão fiscal e dá outras providências, a fim de determinar a disponibilização, em tempo real, de informações pormenorizadas sobre a execução orçamentária e financeira da União, dos Estados, do Distrito Federal e dos Municípios. BRASILIA, 28 maio 2009. Disponível em: http://www.planalto.gov.br/ccivil 03/leis/lcp/Lcp131.htm\#art1. Último acesso em: 10 jan. 2021.

BRASIL. Lei $\mathbf{n}^{\mathbf{0}}$ 12527, de 18 de novembro de 2011. Regula o acesso a informações previsto no inciso XXXIII do art. $5^{\circ}$, no inciso II do $\S 3^{\circ}$ do art. 37 e no $\S 2^{\circ}$ do art. 216 da Constituição Federal; altera a Lei $\mathrm{n}^{\mathrm{o}}$ 8.112, de 11 de dezembro de 1990; revoga a Lei $\mathrm{n}^{\mathrm{o}} 11.111$, de 5 de maio de 2005, e dispositivos da Lei $\mathrm{n}^{\mathrm{o}} 8.159$, de 8 de janeiro de 1991; e dá outras providências. BRASILIA, 19 nov. 2011. Disponível em: http://www.planalto.gov.br/ccivil 03/ ato20112014/2011/lei/112527.htm. Último acesso em: 10 jan. 2021.

CHIGNARD, S. A Brief History of Open Data. ParisTech Review. March 29th, 2013. Dispoível em http://www.paristechreview.com/2013/03/29/brief-history-opendata/. Último acesso em: 12 jan. 2021.

CUNHA, M. A. V. C. D., et al.. Dados abertos nos municípios, estados e governo federal brasileiros. CEAPG-Centro de Estudos em Administração Pública e Governo (EAESP) e GEPI-Grupo de Ensino e Pesquisa em Inovação (Direito SP), volume 3, 2015. Disponível em http://bibliotecadigital.fgv.br/dspace/bitstream/handle/10438/16373/Dados 
Abertos_nos_Munic\%C3\%ADpios_Estados_e_Governo_Federal Brasileiros Volume 3. pdf?sequence=1\&isAllowed=y. Último acesso em: 13 fev. 2020.

DUTRA, C. C.; LOPES, K. M. G. Dados abertos: Uma forma inovadora de transparência. VI Congresso CONSAD de Gestão Pública, Brasília/DF, 2013. Disponível em http:// www.sgc.goias.gov.br/upload/arquivos/2014-04/dados-abertos---uma-forma-inovadora-detransparencial.pdf. Último acesso em: 15 mar. 2020.

EUROPEAN PARLIAMENT AND COUNCIL. Directive 2003/98/EC of 17 November 2003 on the re-use of public sector information. OJ L, 345, 90, 2003.

FREITAS, J. A. C. et al. O ecossistema de dados abertos do governo federal: composição e desafios. Ci.Inf., Brasília, v.47 n.2, p.110-132, maio/ago, 2018. Disponível em http://revista.ibict. br/ciinf/article/view/3952. Último acesso em: 04 abr. 2020.

ISOTANI, S; BITTENCOURT, I. I. Dados abertos conectados: em busca da Web do conhecimento. São Paulo: Novatec Editora, 2015. Disponível em http://pgcl.uenf.br/arquivos/ dadosabertosconectados 011120181613.pdf. Último acesso em: 06 dez. 2020.

KLEIN, R. H.; LUCIANO, E.M.; MACADAR, M. A. Grau de transparência de dados abertos governamentais do site dados.rs.gov.br. E\&G Economia e Gestão, Belo Horizonte, v. 15, n. 41, p. 256-285, 2015. Disponível em https://repositorio.pucrs.br/dspace/bitstream/10923/10052/2/ Grau de Transparencia_de Dados_Abertos_Governamentais do site dados_rs_gov br.pdf. Último acesso em: 04 abr. 2020.

LOPES, B.; AMARAL, J. N.; CALDAS, R. W. (Supervisores e Coord.). Políticas Públicas: conceitos e práticas. Belo Horizonte: SEBRAE/MG, 2008. 48p.

NUGROHO, R. P. et al. A comparison of national open data policies: lessons learned. Transforming Government: People, Process and Policy, v. 9, n. 3, p. 286-308, 2015. Disponível em http://dx.doi.org/10.1108/TG-03-2014-0008. Último acesso em: 05 abr. 2020.

OPEN DATA CHARTER. G8 Open Data Charter. Lough Erne Summit Lough Erne, Northern Ireland, United Kingdom, June 18, 2013. http://www.g8.utoronto.ca/ summit/2013lougherne/lough-erne-open-data.html e https://assets.publishing.service.gov.uk/ government/uploads/system/uploads/attachment data/file/207772/Open Data_Charter.pdf. Último acesso em: 12 jan. 2021.

OPEN GOVERNMENT DATA - OGD. Eight principles of open government data. 2007. Disponível em: https://public.resource.org/8_principles.html. Último acesso em: 12 jan. 2021.

OPEN KNOWLEDGE INTERNATIONAL - OKF. The Open Data Handbook. Disponível em https://opendatahandbook.org/. Último acesso em: 12 jan. 2021.

OPEN GOVERNMENT WORKING GROUP - OGWG. Open government data principles. 2007. Disponível em https://opendefinition.org/od/2.1/en/. Último acesso em: 12 jan. 2021. 
OPEN GOVERNMENT PARTNERSHIP - OGP. Declaração de Governo Aberto. 2011. Disponível em https://www.gov.br/cgu/pt-br/governo-aberto/central-de-conteudo/documentos/ arquivos/declaracao-governo-aberto.pdf. Último acesso em: 12 jan. 2021.

PERNAMBUCO. Estatuto da Empresa Pernambucana de Transporte Coletivo Intermunicipal - EPTI. DECRETO No 48.377, DE 13 DE DEZEMBRO DE 2019. Disponível em https:// legis.alepe.pe.gov.br/texto.aspx?id=48360\&tipo=TEXTOORIGINAL. Último acesso em: 06 dez. 2020.

PINHO, M. D. C. Dados abertos governamentais e democracia digital: o estado da arte e uma aplicação aos portais de dados abertos de seis prefeituras brasileiras. Trabalho de Conclusão de Curso (Bacharelado em Jornalismo) - Universidade Federal da Bahia. 2017. 77 folhas. Disponível em http://repositorio.ufba.br/ri/handle/ri/27014. Último acesso em: 05 jan. 2021.

RUEDIGER, M. A.; MAZOTTE, N. (Ed.). Índice de dados abertos para cidades. Rio de Janeiro: FGV DAPP, 2018. Disponível em http://dapp.fgv.br/publicacao/indice-de-dadosabertos-para-cidades-2018/. Último acesso em: 12 jan. 2021.

SILVA, E.R.A. Continuidades, inovações e retrocessos na evolução do governo digital em Pernambuco. Dissertação (Mestrado em Administração) - Universidade Federal de Pernambuco. CCSA, 2018. 165 folhas. Disponível em https://repositorio.ufpe.br/handle/123456789/33888. Último acesso em: 10 jan. 2021.

SOLAR, M. et al. A Model to Guide the Open Government Data Implementation in Public Agencies. J. UCS, v. 20, n. 11, p. 1564-1582, 2014. Disponível em http://www.jucs.org/ jucs 20 11/a_model to guide/jucs $20 \quad 11 \quad 1564 \quad 1582$ solar.pdf. Último acesso em: 05 abr. 2020.

SUSHA, I.; GRÖNLUND, Å.; JANSSEN, M. Organizational measures to stimulate user engagement with open data. Transforming Government: People, Process and Policy, Vol. 9, n. 2 p. 181 - 206. Disponível em https://doi.org/10.1108/TG-05-2014-0016. Último acesso em: 30 out. 2020.

UNITED STATES. Open government directive. Washington, DC: The White House, 2009. Presidential Document. Memorandum for the Heads of Executive Departments and Agencies. Disponível em: https://www.whitehouse.gov/wp-content/uploads/2019/07/M-19-23.pdf. Último acesso em: 12 jan. 2021.

VELJKOVIĆ, N., BOGDANOVIĆ-DINIĆ, S., STOIMENOV, L. Benchmarking open government: An open data perspective. Government Information Quarterly, vol. 31, n. 2, p. 278-290, 2014. Disponível em https://doi.org/10.1016/j.giq.2013.10.011. Último acesso em: 11 abr. 2020. 